\title{
Evaluating target silencing by short hairpin RNA mediated by the group I intron in cultured mammalian cells
}

\author{
Kousei Noguchi, Yoshio Ishitu and Hiroshi Takaku*
}

\begin{abstract}
Background: The group I intron, a ribozyme that catalyzes its own splicing reactions in the absence of proteins in vitro, is a potential target for rational engineering and attracted our interest due to its potential utility in gene repair using trans-splicing. However, the ribozyme activity of a group I intron appears to be facilitated by RNA chaperones in vivo; therefore, the efficiency of self-splicing could be dependent on the structure around the insert site or the length of the sequence to be inserted. To better understand how ribozyme activity could be modulated in cultured mammalian cells, a group I intron was inserted into a short hairpin RNA (shRNA), and silencing of a reporter gene by the shRNA was estimated to reflect self-splicing activity in vivo. In addition, we appended a theophylline-binding aptamer to the ribozyme to investigate any potential effects caused by a trans-effector.

Results: shRNA-expression vectors in which the loop region of the shRNA was interrupted by an intron were constructed to target firefly luciferase mRNA. There was no remarkable toxicity of the shRNA-expression vectors in Cos cells, and the decrease in luciferase activity was measured as an index of the ribozyme splicing activity. In contrast, the expression of the shRNA through intron splicing was completely abolished in 293T cells, although the silencing induced by the shRNA-expressing vector alone was no different from that in the Cos cells. The splicing efficiency of the aptamer-appended intron also had implications for the potential of trans-factors to differentially promote self-splicing among cultured mammalian cells.
\end{abstract}

Conclusions: Silencing by shRNAs interrupted by a group I intron could be used to monitor self-splicing activity in cultured mammalian cells, and the efficiency of self-splicing appears to be affected by cell-type specific factors, demonstrating the potential effectiveness of a trans-effector.

\section{Background}

The group I intron from Tetrahymena thermophila catalyzes its own excision and ligation of the 5' and 3' exons, meaning that it performs self-splicing without the aid of proteins in vitro [1]. Self-splicing of unusual sequence alignments has been shown to occur in vitro and in vivo in other species, including mammalian species, but group I introns have not been reported in mammals [2]. In addition to self-splicing to ligate two exons that are juxtaposed to a group I intron, several variations on self-splicing have been identified, including trans-splicing, which is used to correct gene sequences with mutations, a promising technique with

\footnotetext{
* Correspondence: hiroshi.takaku@it-chiba.ac.jp

Department of Life and Environmental Science, Chiba Institute of Technology, 2-17-1 Tsudanuma, Narashino, Chiba 275-0016, Japan
}

potential therapeutic application $[3,4]$. Thus, elucidation of the actions of group I introns in vivo would be beneficial $[5,6]$.

The proper structure and the efficient catalysis of group I and group II introns seem to depend on RNA chaperone proteins in vivo [7]. Without the aid of chaperone proteins, misfolding is often triggered from alternative base pairings or in the thermodynamically favored direction. To investigate the splicing activity of the group I intron in cultured mammalian cells, we constructed expression vectors containing a short hairpin RNA (shRNA) interrupted by an intron. The expressed shRNA is converted to a small interfering RNA (siRNA) by the RNase III enzyme DICER, and then it triggers RNA interference (RNAi) in the cells [8-10]. After splicing with the help of chaperone proteins, the resulting
C Biomed Central

() 2011 Noguchi et al; licensee BioMed Central Ltd. This is an Open Access article distributed under the terms of the Creative Commons Attribution License (http://creativecommons.org/licenses/by/2.0), which permits unrestricted use, distribution, and reproduction in any medium, provided the original work is properly cited. 
ligated transcript is predicted to fold back on itself to form a stem-loop structure that produces shRNA, leading to silencing of the targeted reporter gene. The low production level of protein products due to self-splicing limits the utility of group I introns and self-splicing, but in this context, the self-splicing mechanism can be used to produce RNA as the final product [2]. Therefore, this method might provide insight into the mechanism of self-splicing and into the relationship between the self-splicing mechanism and RNA chaperone proteins in vivo.

Two sets of coaxially stacked helices, P4-P6 and P3$\mathrm{P9}$, are the conserved core of group I introns, and the peripheral domains play important roles in stabilizing the functional structures. It has been suggested that P5abc stabilizes the folded structure of group I introns, and the interaction between the tetraloop (L5b) in P5abc and the tetraloop receptor in the P6 stem functions as a clamp to stabilize the conformation [11]. A chaperone protein can substantially rescue the catalytic activity of Tetrahymena introns in which P5abc has been deleted. This chaperone appears to bind the P4-P6 domain and to form a scaffold for the assembly of the P3-P9 domain [12]. Group I ribozymes were successfully activated using an appended aptamer in which the P6 region of the intron from the thymidylate synthase gene in bacteriophage T4 was substituted with an anti-theophylline aptamer [13]. Here, we also attempted substitution of the P6 region of the Tetrahymena intron with an anti-theophylline aptamer so that the specific binding of theophylline to the the group I intron might lead to a conformational change, thus modulating catalysis in a way that is similar to the binding of a chaperone protein. The application of functional RNAs to gene regulation has intriguing possibilities for conditional gene expression and knockdown systems, but the development of these applications is still in progress [14-17].

In the present study, we used silencing of the firefly luciferase gene by shRNAs to evaluate the splicing efficiency of the Tetrahymena thermophila group I intron, which was inserted into the loop region, in a mammalian cultured cell line. In addition, to infer how self-splicing was affected by a trans-effector in mammalian cells, we assessed the effects of an appended aptamer under splicing-competent physiologic conditions.

\section{Results}

Construction of shRNAs containing a microRNA-derived loop and validation of target silencing

The production of shRNA via splicing catalyzed by a Tetrahymena group I intron estimates the splicing efficiency in conjunction with chaperone proteins in mammalian cells (Figure 1A). To this end, shRNAs targeting the firefly luciferase transcript were placed under a

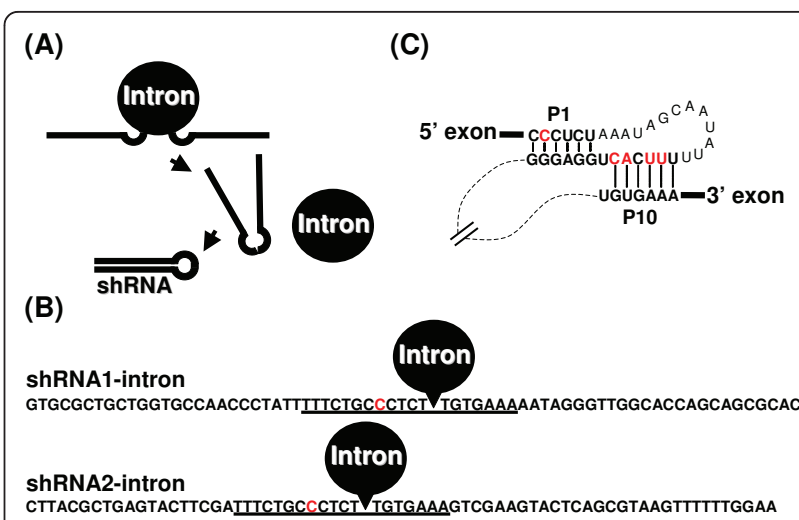

Figure 1 shRNA directed against firefly luciferase and interruption of shRNA by an intron. (A) Schematic representation of the formation of the stem-loop structure through the self-splicing of a group I intron inserted into the loop site. (B) Expression vectors were constructed for shRNAs targeting the "155-173" or "851-875" region of firefly luciferase. The shRNA cassettes were placed under control of the CMV promoter of the pRNA-CMV3.1-Neo vector. The loop portion of the shRNA was from the loop region of hsa-mir-371 with a slight modification. The loop sequences are underlined. The inserted site of the group I intron is illustrated, and the modified nucleotides are shown in red. (C) Schematic representation of the interactions involved in self-splicing including the pairing between the intron and the $5^{\prime}$ and $3^{\prime}$ exon sequences. These pairings, namely the P1 helix (5'exon-intron pairing) and P10 helix (intron-3'exon pairing), are shown in bold, and the modified nucleotides are shown in red.

cytomegalovirus (CMV) promoter, and a group I intron was inserted into the loop region (Figure 1B). Then target silencing by the shRNA was quantitatively analyzed as a reflection of the inserted ribozyme activity. Of note, the transcribed RNA was longer than the shRNA and was expected to be fully expressed under these conditions. Specifically, RNA polymerase II-driven promoters have a variety of expression patterns and are more suitable for achieving a spatio-temporal expression pattern than RNA polymerase III-driven promoters [18]. The sequences of the 5' and 3' exons of the Tetrahymena intron were designed to pair with the intron for efficient and precise splicing, namely the formation of the P1 helix (5' exon-intron pairing) and the formation of the P10 helix (intron-3' exon pairing). To accommodate these characteristics, we searched for a loop portion that was similar to the pairing sequences using a microRNA (miRNA) database, and the hsa-mir-371 sequence with a one-base modification was selected [19]. The one base change of the loop region did not significantly decrease the efficacy of silencing by the shRNA (data not shown). In addition, 4 bases of the intron sequence were modified in conjunction with the loop (Figure 1B, C).

We first constructed two vectors in which the shRNA with the miRNA-derived loop was inserted downstream of the CMV promoter. The first shRNA (shRNA1) 
contained 25 bases of an antisense strand targeting bases 851-875 of the firefly luciferase transcript. The second shRNA (shRNA2) contained 19 bases of the antisense strand targeting bases 155-173 of the firefly luciferase transcript, and it was followed by a poly (U) termination signal based on a report that the poly $(U)$ termination signal could substitute for the polyadenylation signal in the expression of shRNA under the CMV promoter [20]. The efficiency of target silencing was then analyzed by transiently transfecting the shRNA vector in Cos cells with two vectors expressing firefly luciferase and Renilla luciferase, and luciferase activity was analyzed $48 \mathrm{~h}$ and $60 \mathrm{~h}$ after transfection (Figure
2A). In both cases, the reduction of firefly luciferase activity relative to the control vector indicated the efficiency of the shRNAs. In the case of shRNA2, the addition of the poly $(\mathrm{U})$ termination signal appeared to be effective for target silencing because the construct without the poly $(\mathrm{U})$ termination signal (siRNA2- $\Delta \mathrm{U})$ produced less efficient target silencing (Figure 2A).

Interruption of shRNA-mediated target suppression by an intron with cell-type specificity

The loop derived from hsa-mir-371 was sufficient for shRNA-expressing vectors to induce RNAi; therefore, a group I intron was inserted into the middle site of the

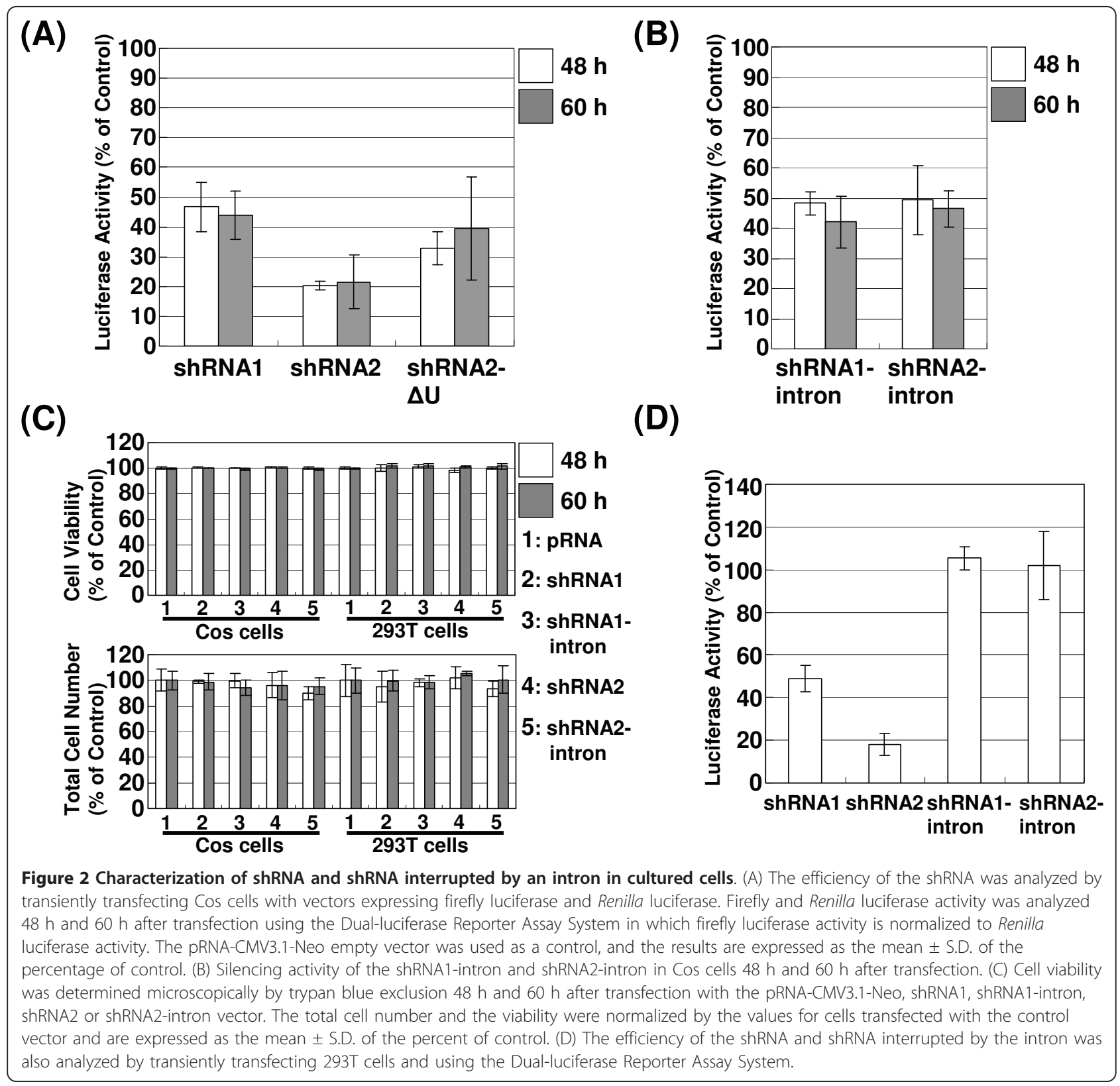


loop sequence (Figure 1B). When the constructed vectors were transiently cotransfected in Cos cells, we observed decreased firefly luciferase activity compared to control vectors (Figure 2B). No significant changes in the shape or viability of the transfected cells were observed (Figure 2C). Furthermore, it is not likely that the group I intron induced the silencing effect via a mechanism such as trans-splicing, that is, direct association before splicing. The sequence corresponding to the 5 ' exon has been used as antisense, and relatively long sequences have been used as the antisense strand for efficient splicing in trans-splicing systems [21]. In the present study, the antisense strand corresponds to the 3' stem of both shRNA1 and shRNA2. Moreover, if it were transcribed, the antisense strand would be followed by an unrelated sequence, specifically the poly (A) tail in the case of the shRNA1-intron and the poly $(\mathrm{U})$ termination signal in the shRNA2-intron. We also repeated this experiment using another routinely used cell line, 293T cells. In stark contrast to the Cos cells, we found no significant repression of luciferase activity, but a similar or even greater silencing effect was observed when the shRNAs without the groupI intron were expressed (Figure 2D). In addition, we observed no significant effect on cell viability (Figure $2 \mathrm{C}$ ). These findings suggest that the suppressive potency of the shRNA-intron is not due to direct activity by the RNase III enzyme DICER and that the splicing efficiency appears to affect the extent of luciferase gene silencing (Figure 2D).

To elaborate on the difference between cell-types, we assessed the expression level of the siRNAs from the shRNA and shRNA-intron vectors. To this end, we designed a stem-loop primer to detect the predicted siRNA and determined that the siRNA was processed from the shRNA precisely as predicted (Figure 3A). We did not observe a significant difference in siRNA expression between Cos cells and 293T cells when the same amount of the shRNA-expressing vector was transfected (Figure 3B, C). However, the amount of siRNAs produced from the shRNA-intron vectors was significantly reduced in 293T cells compared with Cos cells, although the expression level of the intron was not significantly different between the cell types (Figure 3B, C). When we compared the siRNA to intron ratio, as assessed by RT-qPCR, in 293T cells and Cos cells, we confirmed a significant reduction in the level of siRNA (Figure 3D). Taken together, these results suggest that the splicing event and the resulting RNAi appear to occur in a cell-type specific manner, independent of the DICER activity of the cell.

\section{Characterization of sequence-dependent target silencing induced by the shRNA-intron}

To more precisely evaluate whether the observed suppressive effects were specific for the target sequences, we used the psiCHECK-2 vector, which is typically used to check the knockdown efficiency of a target sequence by siRNA or miRNA [22]. Although the psiCHECK-2 vector expresses firefly luciferase in addition to Renilla luciferase, the firefly luciferase gene in this vector has a different sequence from the "155-173" and "851-875" target sequences of the pGL3-Control vector (Figure $4 \mathrm{~A})$. Even under this circumstance, it is plausible that these corresponding sites of psiCHECK-2 vector are also repressed by miRNA interference because unlike siRNA, miRNA does not require perfect complementarity of sequences. Based on the premise that miRNA commonly targets the 3' untranslated region of mRNA and that the silencing effect of siRNA is usually stronger than that of miRNA, the experimental readout would largely reflect the silencing induced by the siRNA [23]. Therefore, we constructed vectors in which the target sequence for each of shRNA1 and shRNA2 was inserted into the 3' UTR of the Renilla luciferase gene of the psiCHECK-2 vector. The constructed vectors, "GL851," which would be targeted by shRNA1, and "GL155", which would be targeted by shRNA2, were transfected, and we assessed the effect of each shRNA-intron on luciferase activity in the transfected cells. As expected, we observed a specific knockdown that corresponded to the inserted target sequences (Figure 4B). Based on these findings, it is plausible that the siRNA was produced from the shRNA-intron and silenced the target in a sequence specific manner.

\section{The modulation of splicing efficiency by an aptamer- ligand interaction}

We assumed that the expression of shRNA from the shRNA-intron is modulated by a cell specific factor at the step at which the inserted intron in the loop region of the shRNA is spliced. Considering that the regional binding of a chaperone protein could promote self-splicing in vivo, we set out to elucidate the effect of a potential trans-effector that binds the regional site in the group I intron in cultured mammalian cells. Intriguingly, the splicing of an aptamer-appended T4 phage group I intron was shown to be modulated in E. coli [13]. To accomplish aptamer-mediated targeting, the P6 region of the Tetrahymena group I intron was replaced with a theophylline-binding aptamer (Figure 5A). The activity of the shRNA-intron-aptamer vector was validated in Cos cells. The substitution of the theophyllinebinding aptamer for the P6 region of the shRNA1-intron led to less efficient silencing, prompting us to investigate the activity of the shRNA1-intron-aptamer when the ligand for the aptamer was present in the culture medium (Figure 5B). Consistent with previous reports of the use of an anti-theophylline aptamer in mammalian cells, the shRNA1-intron-aptamer showed a prominent effect 


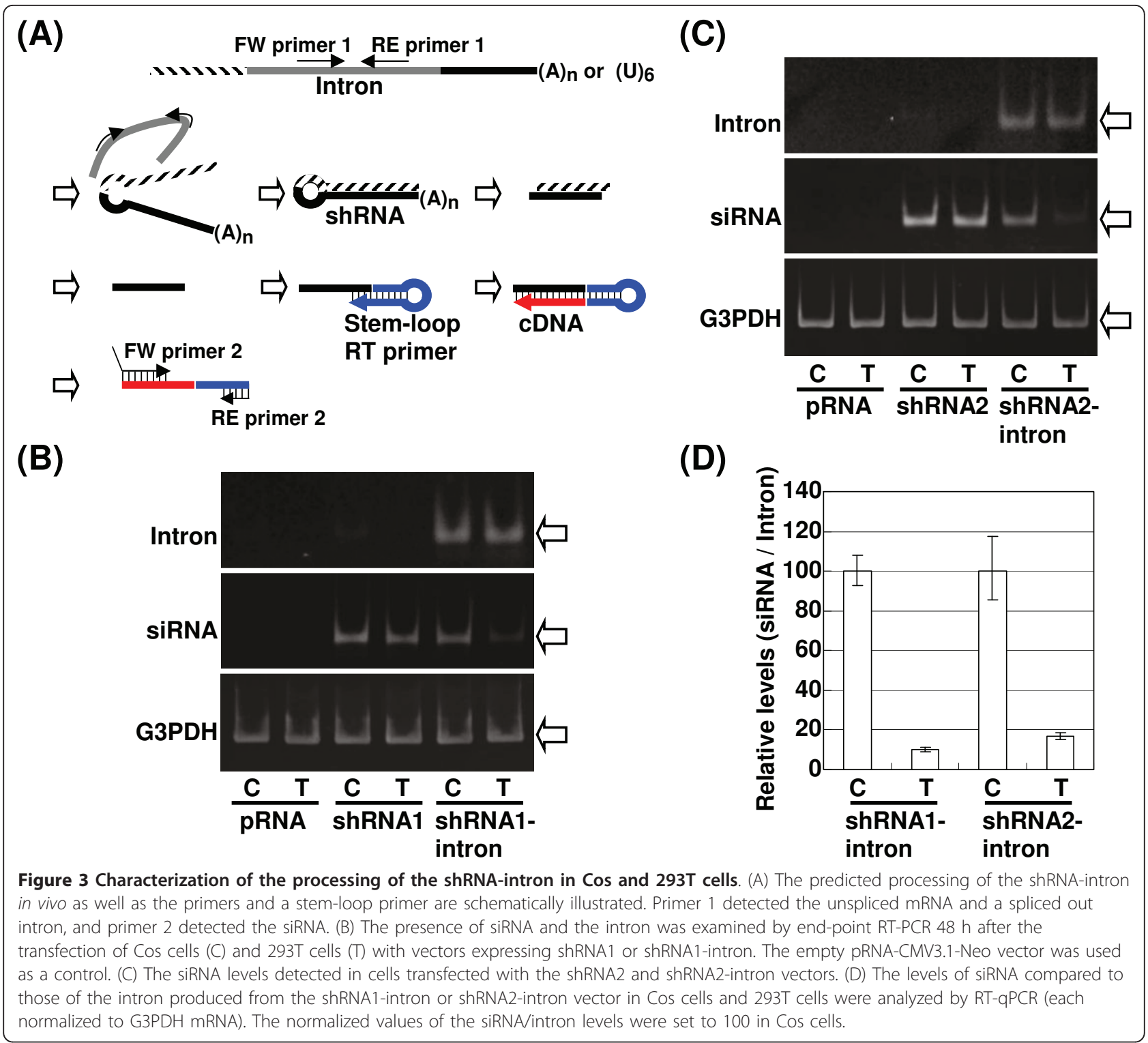

with 8-10 $\mathrm{mM}$ theophylline [24,25]. This concentration of theophylline, however, was highly toxic to the cultured cells. Therefore, we decreased the exposure period and observed a repressive effect similar to that of the shRNA1-intron. The presence of theophylline in the culture medium had no effect on the silencing activity induced by the shRNA1 intron vector (Figure 5B). Adding caffeine $(10 \mathrm{mM})$, which is structurally similar to theophylline but does not bind to the aptamer, did not affect the silencing efficiency (Figure 5C) [26]. Hence, the aptamer-appended group I intron became functional by adding theophylline in a manner analogous to that of the T4 phage group I intron in E. coli [13]. The similar effect, although slightly less robust relative to that of the T4 phage group I intron in E. coli, might be due to the differences in the physiologic conditions between mammalian cells and $E$. coli and in their structure. Nevertheless, we obtained evidence that the intracellular milieu affects the splicing efficiency of different cell lines (Figure 2).

\section{Discussion}

The efficient splicing of group I introns in vivo has been attributed mainly to chaperone proteins, contrasting with splicing in vitro, which occurs in the absence of any proteins [12,27]. In fact, compared to Cos cells, very little target silencing by shRNA-intron vectors was observed in 293T cells, despite the fact that the corresponding shRNA expression vectors had the same or even better effects on target silencing. Given these 


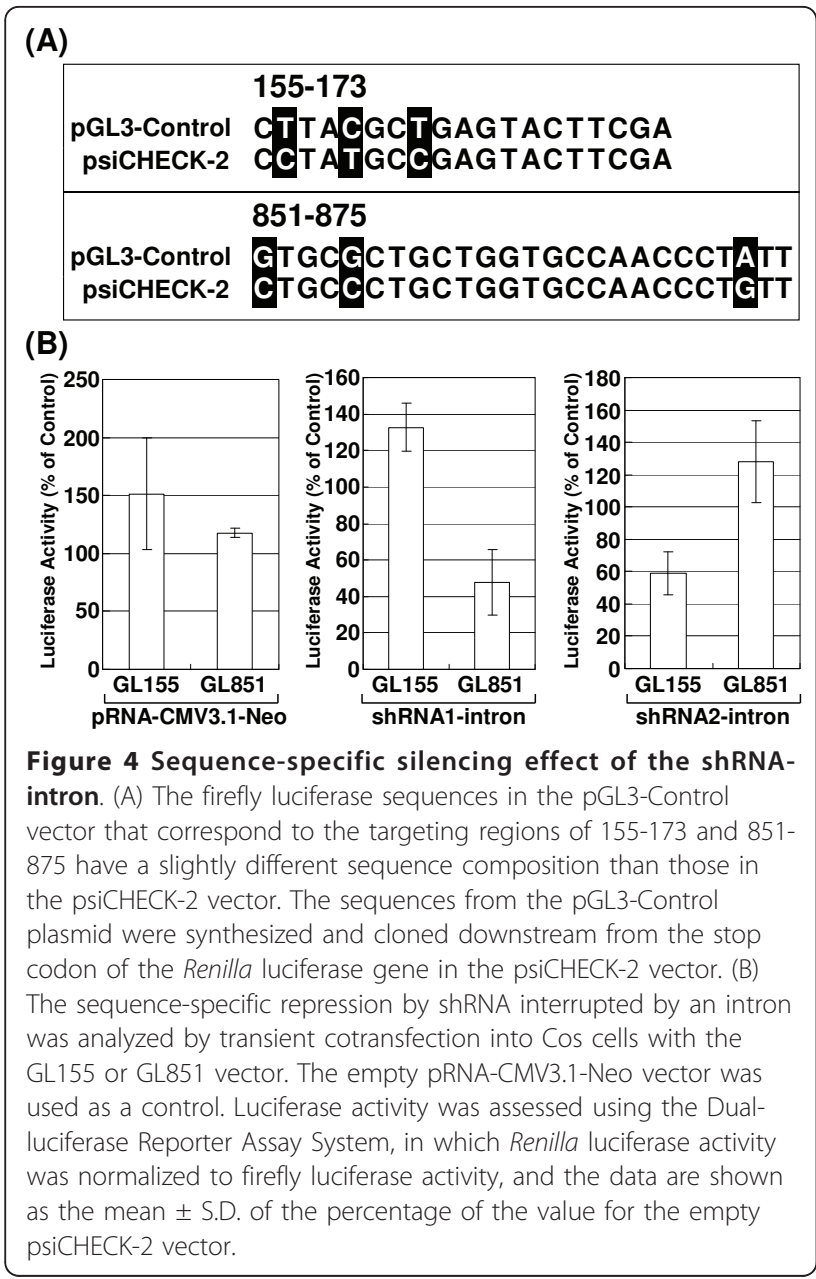

results, target silencing by shRNA-intron vectors might be dependent on the endogenous distribution or the amount of chaperone proteins in different cell types. Alternatively, some substrate-specific chaperone proteins or some inhibitory factors might be involved in the splicing reaction [28-30]. From a therapeutic point of view, it is intriguing that a particular chaperone protein is overexpressed in cancer cells and that could be considered a target for cancer therapy [31]. There is evidence that considerable splicing occurs in 293 cells when group I introns are inserted into a luciferase gene [2]. Therefore, there might be a preferential length, sequence, and structure for in vivo splicing [32]. Thus, several features and obstacles of intron self-splicing are evident when producing shRNA via splicing to analyze RNA chaperone activity in mammalian cells, similar to an "RNA folding trap" [6,33].

The Neurospora crassa mitochondrial tyrosyl-tRNA synthetase (CYT-18 protein) binds the P4-P6 domain of the Tetrahymena group I intron to promote splicing [34]. However this effect has been confirmed only for the synthetases of Neurospora crassa and the closely related fungus Podospora anserina, despite the fact that these proteins share the same basic structure with all bacterial tyrosyl-tRNA synthetases and the Saccharomyces cerevisiae mitochondrial tyrosyl-tRNA synthetase. Theophylline, a small organic molecule, modulates group I intron activity in E. coli and in mammalian cells, and there is a possibility that such a protein could bind and trigger a change in the enzymatic activity of the intron in these species. However, replacement of the P6 region with a theophylline-binding aptamer was not sufficient to completely inactivate self-splicing in Cos cells. Therefore, further replacement or modification could be rationally engineered for finely tuned control. Alternatively, antibiotics, which specifically bind to group I introns in vitro, might also modulate self-splicing; however, many of these compounds remain to be validated in vivo, and others are not as efficient as initially expected [35-39]. Altogether, further understanding of the splicing efficiency of group I introns in physiological conditions might pave the way for applications using modified group I introns and functional RNAs in vivo [40].

\section{Conclusions}

Our results revealed that a miRNA-derived loop could be used to efficiently express shRNAs under a CMV promoter and that the expression of the shRNAs could be mediated by a group I intron inserted into the loop region. The shRNAs expressed via self-splicing of a group I intron affected target silencing in a cell-type specific manner under physiological conditions. In addition, the effect of theophylline when a theophyllinebinding aptamer was embedded in a group I intron supported the feasibility of regulation by a trans-effector as well as the physiological importance of the connection between splicing efficiency and cellular factors.

\section{Methods}

\section{Plasmid construction}

pRNA-CMV3.1-Neo (GenScript Corporation) was used to construct the vectors expressing shRNA and shRNA interrupted by an intron. For the shRNA vectors, a pRNA-CMV3.1-Neo vector was digested with BamHI and HindIII and was ligated to the annealed oligonucleotide. The oligonucleotides used were as follows: shRNA1: 5'-GATCCGTGCGCTGCTGGTGCCAA CCCTATTTTTCTGCCCTCTTGTGAAAAATAGGG TTGGCACCAGCAGCGCACA-3' and 5'-AGCTTGTG CGCTGCTGGTGCCAACCCTATTTTTCACAAGAG GGCAGAAAAATAGGGTTGGCACCAGCAGCGCA CG-3'; and shRNA2: 5'-GATCCTTACGCTGAGTA CTTCGAT TTCTGCTCTCTTGTGAAATCGAAGTACTCAGCGTAAGTTTTTTGGAA-3' and 5'-AGCT 


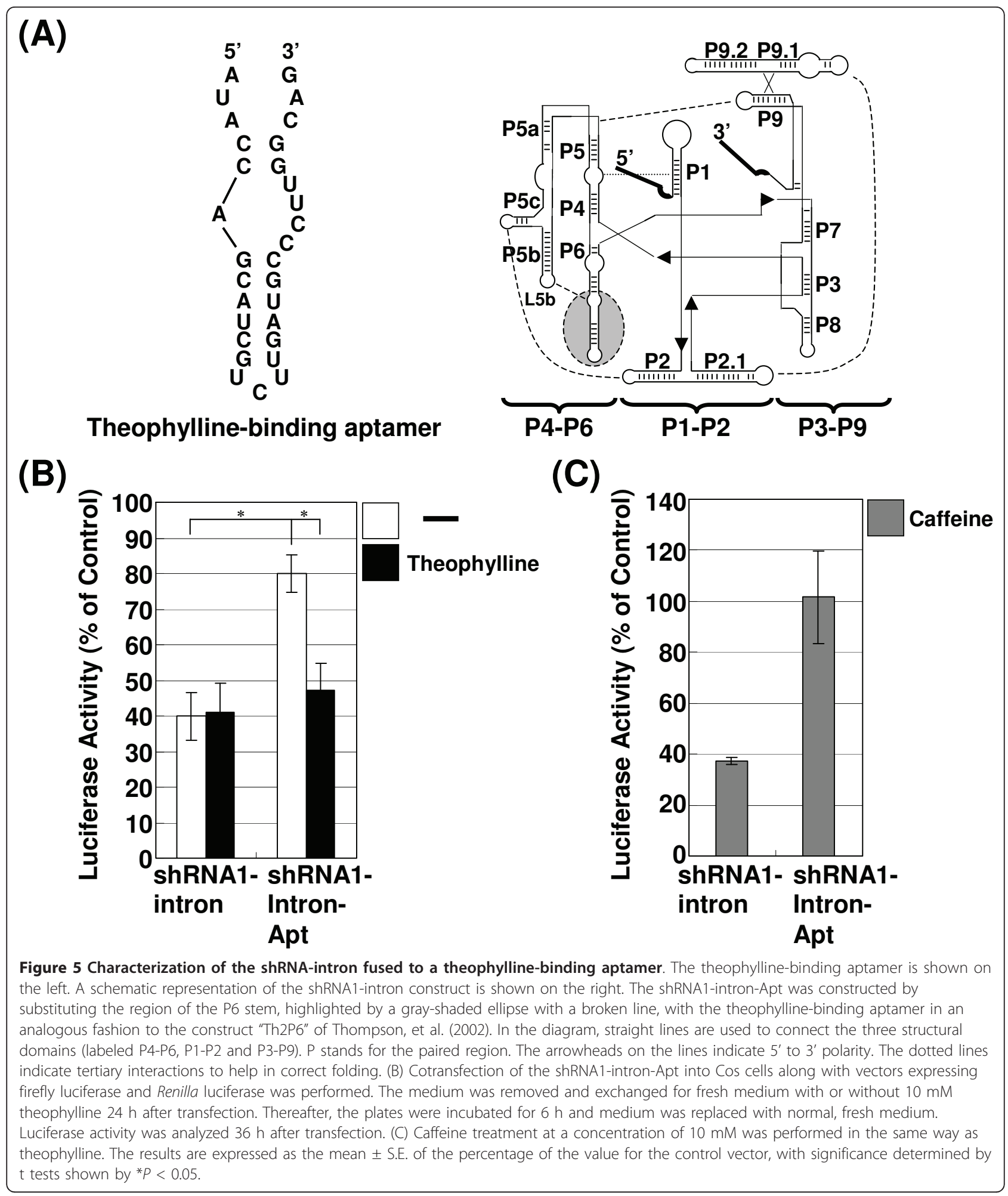


TTCCAAAAAACTTACGCTGAGTACTTCGATTTCA CAAGAGAGCAGAAATCGAAGTACTCAGCGTAAG3'. These vectors were digested with BamHI, treated with mung bean nuclease to remove 5 ' extensions, and self-ligated. To construct the shRNA2 vector, a one base modification of its loop region was performed using the KOD-Plus-Mutagenesis Kit (TOYOBO) according to the manufacturer's instructions. The following primers were used: forward primer: 5'-CCCTCTTGTGAAATCGAAGTACTC-3' and reverse primer: 5'- CAGAAATCGAAGTA CTCAGC-3'. The pTZISVU vector, which contains the group I intron from Tetrahymena thermophila, was digested with EcoRI and HindIII, and its intron-containing fragment was subcloned into the EcoRI-HindIII restriction site of pVAX1 (Invitrogen) to generate pVAX1-intron vector. To construct the shRNA1-intron vector, a vector containing the following sequence at the BamHI-HindIII site of the pRNACMV3.1-Neo vector was first constructed: 5'GATC CGTGCGCTGCTGGTGCCAACCCTATTTT TCTG CCCTCTAAATAGCA AGTATTTTTCACGTG TGAA AAATAGGGTTGGCACCAGCAGCGCACA-3'. This vector (preshRNA1-intron) was then digested with PmlI, and the PCR-amplified intron was ligated. The following primers were used: forward primer: 5'-TGGAGGGAAAAGTTATCAGGC-3' and reverse primer: 5'GAGTACTCCAAAACTAATCAATATACT-3'. To construct the vector expressing the shRNA2-intron, the vector containing the following sequence at the BamHIHindIII site of the pRNA-CMV3.1-Neo vector was first constructed: 5'-GATCCTTACGCTGAGTACTTCGA TTTCTGCCCTCTAAATAGCAATATTTTTCACGT GTGAAATCGAAGTACTCAGCGTAAGTTTTTTGGAA-3'. This vector (preshRNA2-intron) was digested with PmlI, and the PCR-amplified intron was ligated as well in the shRNA1-intron. To construct the shRNA1intron-Apt vector, the following oligonucleotides were annealed and ligated into the pVAX1-intron digested with AhdI and EcoNI: 5'-TAGTCTGTGAACTGCATC CATATCCTGCCAAGGGCATCAAGACGATGCTGGTATGACTTGGCTGCGTGGTTAGGACCATGTCCGT CAGCTTATTACCATACCCTTT-3' and 5'-CAAAGG GTATGGTAATAAGCTGACGGACATGGTCCTAACCACGCAGCCAA GTCATACCAGCATCGTCTTGAT GCCCTTGGCAGGATATGGATGCAGTTCACAGACTAA-3'. The resulting vector was PCR-amplified with the following primers then ligated with the preshRNA1intron digested with PmlI: forward primer: 5'-TGGA GGGAAAAGTTATCAGG C-3' and reverse primer: 5'GAGTACTCCAAAACTAATCAATATACT-3'. The GL851 and GL155 vectors were constructed by inserting the annealed oligonucleotide into the PmeI site of the psiCHECK-2 Vector (Promega). All subcloned sequences were verified by DNA sequencing.

\section{Cell culture and transfections}

Cos cells and 293T cells were maintained in Dulbecco's modified Eagle's medium (DMEM) supplemented with $10 \%$ fetal bovine serum, $100 \mathrm{U} / \mathrm{ml}$ penicillin, and 100 $\mu \mathrm{g} / \mathrm{ml}$ streptomycin in a $5 \% \mathrm{CO} 2$-humidified incubator at $37^{\circ} \mathrm{C}$. One day before transfection, the cells were trypsinized and seeded into 24-well plates at a density of 2-3 $\times 10^{4}$ cells/well. Cotransfection was performed using FuGENE6 (Roche Diagnostics) according to the manufacturer's instructions. Specifically, 1 ng of pGL3-Control vector (Promega) and $5 \mathrm{ng}$ of phRG-TK vector (Promega) for Cos cells or $5 \mathrm{ng}$ of pGL3-Control vector and $1 \mathrm{ng}$ of phRG-TK vector for $293 \mathrm{~T}$ cells were cotransfected with $400 \mathrm{ng}$ of the shRNA vector, shRNAintron vector, or shRNA-intron-Apt vector using $1 \mu \mathrm{l}$ of the FuGENE6 reagent per well. For the theophylline or caffeine treatment, the cells were incubated in a $5 \%$ $\mathrm{CO} 2$-humidified incubator at $37^{\circ} \mathrm{C}$ in DMEM supplemented with $10 \mathrm{mM}$ theophylline or caffeine beginning $24 \mathrm{~h}$ after transfection. After $6 \mathrm{~h}$, the medium containing theophylline or caffeine was again exchanged to normal DMEM. After incubation with cell culture medium for the indicated times, the cells were lysed in a passive lysis buffer (Promega). Firefly and Renilla luciferase signals were measured using the Dual-Luciferase ${ }^{\circledR}$ Reporter Assay System (Promega).

\section{Determination of cell number and viability}

A total of $2 \times 10^{4}$ cells/well of Cos cells and $3 \times 10^{4}$ cells/well of 293T cells were seeded into 24-well plates. After incubation for $24 \mathrm{~h}, 400 \mathrm{ng}$ of the vector, shRNA1, shRNA1-intron, shRNA2 or shRNA2-intron was transfected. At the same time, the cells were also transfected with the pRNA-CMV3.1-Neo empty vector as a control. After incubation for $48 \mathrm{~h}$ and $60 \mathrm{~h}$, the total cell number and viability were determined using a standard trypan blue membrane permeability assay. Live and dead cells were stained with trypan blue, the total cell numbers were counted on a conventional hemocytometer, and the percent viability was calculated as the number of live cells/total number of cells $\times 100$. The total cell number and the viability of the cells transfected with the control vector were set at $100 \%$. The number and viability of each type of transfected cell are expressed as the mean \pm S.D. of the percent of control.

\section{RNA extraction, reverse transcription (RT), and real-time qPCR}

Total RNA samples from Cos cells and 293T cells were obtained $48 \mathrm{~h}$ after transfection using the mirVana miRNA Isolation Kit (Ambion) according to the manufacturer's protocol. For detection of siRNAs, stem-loop RT primers were designed, and pulsed reverse transcription was performed $[41,42]$. The stem-loop RT primer 
for the shRNA1 and shRNA1-intron had the following sequence: 5'-GTCGTATCCAGTGCAGGGTCCGAG GTATTCGCACTGGATACG ACGCTGCT-3'. The sequence for the shRNA2 and shRNA2-intron stemloop RT primer was as follows: 5'-GTCGTATCCAGTGCAGGGTCCGAGGTATTCGCACTGGATACGACAACTT A-3'. For detection of the intron and G3PDH mRNA, cDNAs were synthesized with the ReverTra Ace qPCR RT Kit (TOYOBO). qPCR analysis was performed using specific primer pairs and the THUNDERBIRD qPCR Mix (TOYOBO). The results were evaluated by the comparative threshold cycle method [43]. The following primers were used: for the siRNAs produced from the shRNA1 and shRNA1-intron vectors, forward primer: 5'-TCGCGAATAGG GTTGGCACC-3' and reverse primer: 5'-GTGCAGGGTCCGAGGT-3'; for the siRNAs produced from the shRNA2 and shRNA2-intron vectors, forward primer: 5'-TCGCGTCGAAG TACTCAGCG-3' and reverse primer: 5'-GTGCAGGGTCCGAGGT-3'; for the intron, forward primer: 5'-GCCTT GCAAAGGGTATGGTAAT-3' and reverse primer: 5'TAGGACTTGGCTG CGTGGTT-3'; and for G3PDH, forward primer: 5'-AACAGCGACACCCACTCCTC-3' and reverse primer: 5'-TCCACCACCCTGTTGCTGTA3'. The end-point PCR products were electrophoresed on a $15 \%$ polyacrylamide gel and were stained in ethidium bromide.

\section{Acknowledgements}

The pTZISVU vector was kindly provided by Dr. Tan Inoue, Graduate School of Biostudies, Kyoto University, Japan. This work was supported in part by a Grant-in-Aid for High Technology Research from the Ministry of Education, Science, Sports, and Culture, Japan; by a Grant-in-Aid for AIDS research from the Ministry of Health, Labor and Welfare, Japan; and by a Grant of Strategic Research Foundation Grant-aided Project for Private Universities from the

Ministry of Education, Culture, Sport, Science, and Technology, Japan (MEXT).

\section{Authors' contributions}

KN designed and conducted most of the experimental work, compiled and analyzed the data, and drafted the manuscript. Yl participated in constructing the expression vectors and in conducting the cotransfection experiments. HT provided funding and finalized the manuscript. All authors approved the final manuscript.

Received: 16 April 2010 Accepted: 25 July 2011 Published: 25 July 2011

\section{References}

1. Cech TR: Self-splicing of group I introns. Annu Rev Biochem 1990, 59:543-568.

2. Hagen M, Cech TR: Self-splicing of the Tetrahymena intron from mRNA in mammalian cells. EMBO J 1999, 18:6491-6500.

3. Sullenger BA, Cech TR: Ribozyme-mediated repair of defective mRNA by targeted, trans-splicing. Nature 1994, 371:619-622.

4. Fiskaa T, Birgisdottir AB: RNA reprogramming and repair based on transsplicing group I ribozymes. N Biotechnol 2010, 27:194-203.

5. Semrad K, Schroeder R: A ribosomal function is necessary for efficient splicing of the T4 phage thymidylate synthase intron in vivo. Genes Dev 1998, 12:1327-1337.

6. Clodi E, Semrad K, Schroeder R: Assaying RNA chaperone activity in vivo using a novel RNA folding trap. EMBO J 1999, 18:3776-3782.
7. Russell R: RNA misfolding and the action of chaperones. Front Biosci 2008, 13:1-20.

8. Elbashir SM, Harborth J, Lendeckel W, Yalcin A, Weber K, Tuschl T: Duplexes of 21-nucleotide RNAs mediate RNA interference in cultured mammalian cells. Nature 2001, 411:494-498.

9. Brummelkamp TR, Bernards R, Agami R: A system for stable expression of short interfering RNAs in mammalian cells. Science 2002, 296:550-553.

10. Paddison PJ, Silva JM, Conklin DS, Schlabach M, Li M, Aruleba S, Balija V, O'Shaughnessy A, Gnoj L, Scobie K, Chang K, Westbrook T, Cleary M, Sachidanandam R, McCombie WR, Elledge SJ, Hannon GJ: A resource for large-scale RNA-interference-based screens in mammals. Nature 2004, 428:427-431.

11. Cate JH, Gooding AR, Podell E, Zhou K, Golden BL, Kundrot CE, Cech TR, Doudna JA: Crystal structure of a group I ribozyme domain: principles of RNA packing. Science 1996, 273:1678-1685.

12. Mohr G, Caprara MG, Guo Q, Lambowitz AM: A tyrosyl-tRNA synthetase can function similarly to an RNA structure in the Tetrahymena ribozyme. Nature 1994, 370:147-150.

13. Thompson KM, Syrett HA, Knudsen SM, Ellington AD: Group I aptazymes as genetic regulatory switches. BMC Biotechnol 2002, 2:21.

14. Beisel CL, Bayer TS, Hoff KG, Smolke CD: Model-guided design of ligandregulated RNAi for programmable control of gene expression. Mol Syst Biol 2008, 224.

15. Kumar D, An Cl, Yokobayashi Y: Conditional RNA interference mediated by allosteric ribozyme. J Am Chem Soc 2009, 131:13906-13907.

16. Lee SK, Kumar P: Conditional RNAi: Towards a silent gene therapy. Adv Drug Deliv Rev 2009, 61:650-664.

17. Tuleuova N, An Cl, Ramanculov E, Revzin A, Yokobayashi Y: Modulating endogenous gene expression of mammalian cells via RNA-small molecule interaction. Biochem Biophys Res Commun 2008, 376:169-173.

18. Zhou $H, X i a X G, X u Z$ : An RNA polymerase II construct synthesizes shorthairpin RNA with a quantitative indicator and mediates highly efficient RNAi. Nucleic Acids Res 2005, 33:e62.

19. Griffiths-Jones S: miRBase: microRNA sequences and annotation. Curr Protoc Bioinformatics 2010, 9:1-10.

20. Song J, Pang S, Lu Y, Chiu R: Poly(U) and polyadenylation termination signals are interchangeable for terminating the expression of shRNA from a pol II promoter. Biochem Biophys Res Commun 2004, 323:573-578.

21. Köhler U, Ayre BG, Goodman HM, Haseloff J: Trans-splicing ribozymes for targeted gene delivery. J Mol Biol 1999, 285:1935-1950.

22. Landthaler M, Yalcin A, Tuschl T: The Human DiGeorge Syndrome Critical Region Gene 8 and Its D. melanogaster Homolog Are Required for miRNA Biogenesis. Curr Biol 2004, 12:2162-2167.

23. Bartel DP: MicroRNAs: target recognition and regulatory functions. Cell 2009, 136:215-33.

24. An Cl, Trinh VB, Yokobayashi Y: Artificial control of gene expression in mammalian cells by modulating RNA interference through aptamersmall molecule interaction. RNA 2006, 12:710-716.

25. Kim DS, Gusti V, Dery KJ, Gaur RK: Ligand-induced sequestering of branchpoint sequence allows conditional control of splicing. BMC Mol Biol 2008, 9:23.

26. Jenison RD, Gill SC, Pardi A, Polisky B: High-resolution molecular discrimination by RNA. Science 1994, 263:1425-1429.

27. Tijerina $P$, Bhaskaran $H$, Russell R: Nonspecific binding to structured RNA and preferential unwinding of an exposed helix by the CYT-19 protein, a DEAD-box RNA chaperone. Proc Natl Acad Sci USA 2006, 103:16698-16703.

28. Ameres SL, Shcherbakov D, Nikonova E, Piendl W, Schroeder R, Semrad K: RNA chaperone activity of $\mathrm{L} 1$ ribosomal proteins: phylogenetic conservation and splicing inhibition. Nucleic Acids Res 2007, 35:3752-3763.

29. Belisova A, Semrad K, Mayer O, Kocian G, Waigmann E, Schroeder R, Steiner G: RNA chaperone activity of protein components of human Ro RNPs. RNA 2005, 11:1084-1094.

30. Halls C, Mohr S, Del Campo M, Yang Q, Jankowsky E, Lambowitz AM: Involvement of DEAD-box proteins in group I and group II intron splicing. Biochemical characterization of Mss116p, ATP hydrolysisdependent and -independent mechanisms, and general RNA chaperone activity. J Mol Biol 2007, 365:835-855.

31. Soo ET, Yip GW, Lwin ZM, Kumar SD, Bay BH: Heat shock proteins as novel therapeutic targets in cancer. In Vivo 2008, 22:311-315.

32. Long $M B$, Sullenger BA: Evaluating group I intron catalytic efficiency in mammalian cells. Mol Cell Biol 1999, 19:6479-6487. 
33. Prenninger S, Schroeder R, Semrad K: Assaying RNA chaperone activity in vivo in bacteria using a ribozyme folding trap. Nat Protoc 2006, 1:1273-1277.

34. Paukstelis PJ, Lambowitz AM: Identification and evolution of fungal mitochondrial tyrosyl-tRNA synthetases with group I intron splicing activity. Proc Natl Acad Sci USA 2008, 105:6010-6015.

35. Labuda LP, Pushechnikov A, Disney MD: Small molecule microarrays of RNA-focused peptoids help identify inhibitors of a pathogenic group I intron. ACS Chem Biol 2009, 4: 299-307.

36. Mei HY, Cui M, Lemrow SM, Czarnik AW: Discovery of selective, smallmolecule inhibitors of RNA complexes-II. Self-splicing group I intron ribozyme. Bioorg Med Chem 1997, 5:1185-1195.

37. Yen L, Magnier M, Weissleder R, Stockwell BR, Mulligan RC: Identification of inhibitors of ribozyme self-cleavage in mammalian cells via highthroughput screening of chemical libraries. RNA 2006, 12:797-806.

38. Yen L, Svendsen J, Lee JS, Gray JT, Magnier M, Baba T, D'Amato RJ, Mulligan RC: Exogenous control of mammalian gene expression through modulation of RNA self-cleavage. Nature 2004, 431:471-476.

39. Zhang Y, Li Z, Pilch DS, Leibowitz MJ: Pentamidine inhibits catalytic activity of group I intron Ca.LSU by altering RNA folding. Nucleic Acids Res 2002, 30:2961-2971.

40. Hasegawa S, Jackson WC, Tsien RY, Rao J: Imaging Tetrahymena ribozyme splicing activity in single live mammalian cells. Proc Natl Acad Sci USA 2003, 100:14892-14896.

41. Chen C, Ridzon DA, Broomer AJ, Zhou Z, Lee DH, Nguyen JT, Barbisin M, Xu NL, Mahuvakar VR, Andersen MR, Lao KQ, Livak K, Guegler KJ: Real-time quantification of microRNAs by stem-loop RT-PCR. Nucleic Acids Res 2005, 33:e179.

42. Varkonyi-Gasic E, Wu R, Wood M, Walton EF, Hellens RP: Protocol: a highly sensitive RT-PCR method for detection and quantification of microRNAs. Plant Methods 2007, 3:12.

43. Schmittgen TD, Livak KJ: Analyzing real-time PCR data by the comparative C(T) method. Nat Protoc 2008, 3:1101-1108.

doi:10.1186/1472-6750-11-79

Cite this article as: Noguchi et al: Evaluating target silencing by short hairpin RNA mediated by the group I intron in cultured mammalian cells. BMC Biotechnology 2011 11:79.

\section{Submit your next manuscript to BioMed Central and take full advantage of:}

- Convenient online submission

- Thorough peer review

- No space constraints or color figure charges

- Immediate publication on acceptance

- Inclusion in PubMed, CAS, Scopus and Google Scholar

- Research which is freely available for redistribution

Submit your manuscript at www.biomedcentral.com/submit 